\title{
Sjef Houppermans
}

\section{À LA RECHERCHE DES IMAGES PERDUES}

Proust et Heuet

Les adaptations en bd des grands chefs-d'œuvre de la littérature constituent une catégorie à part. Stéphane Heuet s'est astreint à 'stripper' La recherche de Marcel Proust. Nous examinerons dans quelle mesure on peut parler de fidélité à l'original et, d'autre part, de quelle manière l'auteur a su exploiter les moyens spécifiques de son média.

RELIEF 2 (3), 2008 - ISSN: 1873-5045. P398-423

http://www.revue-relief.org

URN:NBN:NL:UI:10-1-100016

Igitur, Utrecht Publishing \& Archiving Services

(c) The author keeps the copyright of this article

La bande dessinée - comme d'ailleurs le cinéma - a eu dès le début une relation intense avec les 'grands textes littéraires'. Malgré la diversification qui s'est manifestée surtout depuis 1970 on peut constater un retour régulier au commerce avec les chefs-d'œuvres. Parmi d'autres intentions il est possible de distinguer deux orientations majeures : l'exploitation et la serviabilité. La première tendance choisit surtout les textes qui semblent se prêter tout particulièrement à une mise en images plus ou moins spectaculaire, les romans d'aventures ou les histoires de suspense tels différents textes de Stevenson et de Poe. La seconde manière se veut volontiers pédagogique, prétendant faciliter l'accès à des œuvres réputées 'difficiles' - genre Shakespeare et Tolstoï. En outre on peut remarquer une continuation de la tradition séculaire de la Bible illustrée où à l'Écriture 
Sainte se joignent les classiques de la mythologie et les légendes 'éternelles'. D'autre part il n'est pas toujours aisé de déterminer les limites entre les éditions illustrées et les transformations en bande dessinée. Ainsi la magnifique version du Voyage au bout de la nuit que Tardi a créée est considérée parfois comme véritable bande dessinée. Récemment l'éditeur Delcourt a lancé une nouvelle série Ex-libris présentée de la manière suivante: "Première collection dédiée aux adaptations de grands classiques de la littérature française et étrangère. Cette collection s'attache à toujours respecter les textes et mises en scène de l'œuvre. » On trouvera dans cette collection des titres attendus comme Les aventures de Tom Sawyer ou encore des textes de Gauthier, Hugo, Verne et Dumas, mais aussi Kafka et Oscar Wilde. Le moins qu'on puisse dire c'est qu'il y a un renouveau d'intérêt pour ce genre de créations.

Chez le même éditeur Delcourt existait déjà une autre adaptation d'un texte littéraire - qui probablement a servi de stimulateur pour la nouvelle collection, mais qui ressortit sous la catégorie 'hors collection' - à savoir la version de la Recherche de Proust dessinée par Stéphane Heuet. À l'heure actuelle il s'agit de quatre albums: Combray (1998), À l'ombre des jeunes filles en fleurs 1 et 2 (2000, 2002), Un Amour de Swannn 1 (2006, alors qu'en automne 2008 sera publié Un Amour de Swannn 2). C'est un succès commercial qui a été suivi par sa traduction en plusieurs langues étrangères. Parmi les 'proustiens' les opinions divergent mais généralement l'accueil a été plutôt positif.

\section{Forme extérieure}

La forme des albums ${ }^{1}$ est traditionnelle $(32$ par $23 \mathrm{~cm}$ ) contenant chaque fois 48 pages numérotées, exception faite de Combray qui en compte 72 . Le cadre de la couverture est 'classique', 'palladien' d'aspect, monumental, alors que la couleur du fond est ce teint ambré qui rappelle les photos d'autrefois. On peut avoir la suggestion d'une scène de théâtre à l'italienne où à l'intérieur du cadre constitué par les bords extérieurs du livre un deuxième cadre à doubles traits (mordorés) suggérerait les rideaux, alors que l'image centrale entourée d'un trait noir $(15 \times 19)$ visualise la scène 
proprement dite. Ainsi est souligné le côté majestueux du texte proustien mais d'autre part est accentué de la sorte la dimension théâtrale de l'œuvre. Notons encore que pour chacun des albums un personnage se trouve entre les deux cadres intérieurs. Pour Combray c'est le 'je' adulte qui se rapproche du je-narrateur. En principe ce je-narrateur bouge en effet entre les cadres de la Recherche en indiquant ainsi l'intention profonde qui est de retrouver le temps perdu, ou plus précisément une unité morcelée par la progression du temps. Sur la couverture de Combray ce personnage sort du cadre intérieur sur une ligne où sous un ciel identique se réunissent et s'amalgament à la Magritte le décor parisien et le paysage de Combray. L'homme est vêtu d'un lourd manteau d'hiver et son visage est caché dans la fourrure (la couverture exhibe ainsi la dimension 'couverte', cachée). On peut supposer que tout en tournant le dos à la scène des souvenirs, il nous y fait entrer à la rencontre de l'enfant qu'il a été. 


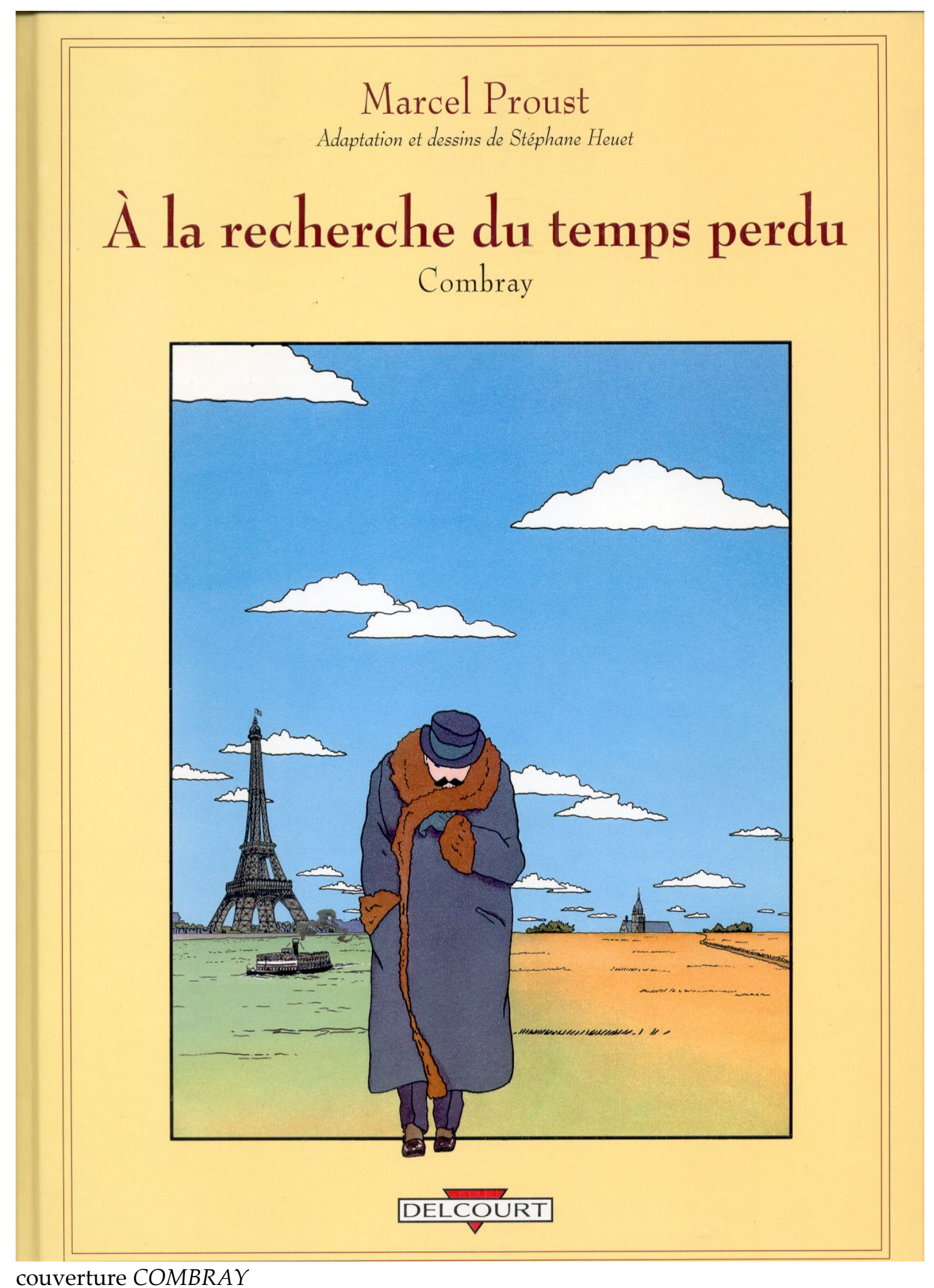


Pour Les Jeunes Filles 1 on trouve la même dichotomie: l'adolescent qui figure dans l'histoire à l'intérieur du recueil se redouble dans le je adulte qui se trouve à la limite d'une longue jetée-promenade dont la perspective suggère l'envol des souvenirs. Dans Les Jeunes Filles 2 la couverture combine deux événements majeurs: la visite de l'atelier du peintre Elstir qui sera le grand exemple au niveau artistique et esthétique, et d'autre part l'apparition de la jeune sportive qu'est Albertine Simonet, future passion principale de Marcel. Ici le jeu avec les cadres (et donc avec les conventions de la bd) est particulièrement sophistiqué : c'est le genre du 'cabinet d'amateur' où sont exposées les tableaux marins d'Elstir qui est justement en train de peindre un lever du soleil sur la mer. Albertine surgit dans le cadre de la fenêtre ouvrant sur l'extérieur et se trouve sur une même ligne avec le jeune visiteur à l'autre bout de l'atelier. Pourtant ce dernier ne se trouve pas intégralement dans l'image ; il sort du cadre par le derrière étant assis dans une pose ambivalente sur un tabouret à vis et tenant à la main un tableau datant d'une autre période d'Elstir (représentant Odette de Crécy en costume d'actrice, relevant donc d'une autre dimension fictionnelle et existentielle). Elstir est le peintre des métaphores picturales dans ses rencontres de la terre et de la mer: c'est vers cet art de la métaphore que va évoluer le narrateur, mais ce sera aux dépens des relations amoureuses aboutissant à la perte de cette Albertine avec qui il n'a peut-être jamais été dans «le même cadre». Dans cet univers de doubles et de redoublements présence et représentation se confondent - ce que souligne encore le jeu des ombres (d'Albertine, des pinceaux sur la table, du pinceau sur la toile, du tabouret).

Un amour de Swann finalement nous montre Swann entrant-sortant quant au cadre intérieur qui représente la maison d'Odette rue la Pérouse vue de l'extérieur, avec comme cadre supplémentaire la fenêtre illuminée derrière laquelle se profile en ombres chinoises un couple dont l'homme offre un collier à la femme (des rideaux jaune d'œuf surplombant la vision). Fantasmagorie ou hallucination d'un Swann dont la jalousie colore toute observation (union préfigurée de Forcheville et d'Odette), Swann qui concrètement, matériellement, dimensionnellement est l'exclu. " Elle n'était pas de son genre ». Nous voyons ainsi que là où il n'y pas encore trace du 
texte proustien les images racontent d'ores et déjà l'essentiel de l'histoire (pour qui connaît le texte proustien, mais encore pour celui qui referme l'album avant d'entamer un second parcours).

Et il y a plus. La quatrième de couverture montre également une image encadrée où l'on découvre l'auteur (Proust) écrivant dans son lit entouré de plusieurs cahiers, alors qu'on remarque également à côté de lui un bol fumant dont les émanations montent au dessus de la lampe de chevet. Ici un fragment de texte coiffe l'image, présenté nettement comme citation : «Et comme un aviateur qui a jusque-là péniblement roulé à terre, "décollant" brusquement, je m'élevais lentement vers les hauteurs silencieuses du souvenir. »Cette sensation a d'ailleurs lieu quand il traverse Paris pour aller à la matinée chez le Prince de Guermantes dans Le Temps retrouvé et le lien suggéré avec le breuvage provient de l'inspiration du dessinateur. On s'aperçoit que le nuage de vapeur trace la forme d'un phylactère ou d'une bulle étirée de bande dessinée avec l'extrémité fléchée qui pointe vers le haut : la voix vient d'un ailleurs mystérieux.

En continuant à feuilleter l'album on découvre que l'entrée se fait dans les quatre cas par une double page dessinée.

Pour Combray nous voyons le petit garçon - Marcel - devant une grande grille qui ouvre sur un domaine à la campagne. Cette même double page revient à la fin avant la quatrième de couverture. Ainsi est figurée l'attente de l'enfant (sur cette image il n'a pas de bouche en véritable infans) : visiblement il est devant la demeure des Guermantes, sur le seuil de l'inconnu et du merveilleux. Pareillement le lecteur est invité à entrer dans l'album, ce qui crée une vraie connivence des niveaux.

La pratique de la double page identique à la fin et au début du recueil se retrouve pour Les Jeunes Filles - et c'est même une image toute pareille pour les quatre occurrences. On y voit encore Marcel en jeune adolescent qui descend une dune pour se diriger vers la plage. On n'aperçoit pas encore cette plage, ni la mer. Autre entrée donc, autre approche, des jeunes filles peut-on supposer, de la mer, de tout ce qui se trouve sur cette plage, autre invitation au lecteur. La solitude de la personne souligne l'ouverture de l'attente, la virtualité des choses à venir, l'offre de cette petite figure humaine, sous ce vaste ciel où circulent juste quelques mouettes, focalisation du désir. 
La double page dans Un Amour de Swann présente une vaste vue de Paris, du cœur de la ville, allant de Notre Dame vue de derrière à l'Ile Saint-Louis où habite Swann, Quai d'Orléans. Bien sûr les quais se trouvent dans l'état où ils ont dû être vers 1875, l'époque de l'affaire entre Swann et Odette. Là encore un paysage qui s'ouvre devant le spectateur de ces lieux.

Parmi ces données paratextuelles qui dans le cas d'une bd jouent un rôle généralement plus important que pour d'autres textes, on peut encore mentionner la page de titre où figure comme image la fameuse lanterne magique génératrice de projections merveilleuses. On aperçoit d'ailleurs que de son sommet sort encore un nuage de vapeur pareil à celui qui monte du bol de tisane. Ici l'extrémité de cette pseudo-bulle se dirige pourtant non vers le haut mais vers l'intérieur de l'album et à côté de l'appareil on voit quelques planches qui annoncent le récit de Geneviève de Brabant, histoire noyau de toutes les affaires d'amour (maternel) ${ }^{2}$. À l'endroit en question se trouve dans Jeunes Filles 1 une tente de plage et dans Jeunes Filles 2 un chevalet de peintre posé sur le sable accompagné d'un chapeau qui s'envole (autre manière de combiner art et désir). Pour Swann enfin il s'agit de ses attributs d'homme du monde élégant: haut de forme, badine, gant et écharpe. Il s'en pare pour sortir dans le monde et nous invite à le suivre (comme dans le film de Schlöndorf).

\section{Projet global}

Si donc on peut parler d'une vraie recherche dans ces éléments paratextuels, regardons maintenant avant d'entrer en détail comment a été conçu le projet total. Lisiblement le but a été de se mettre d'abord au service du texte proustien. Il s'agit plutôt d'un livre illustré que d'une bande dessinée qui s'inspire de la Recherche suivant ses propres principes et ses propres possibilités (médiales, techniques, compositrices). La première conséquence en est l'emploi des citations: chaque page des albums ou presque est truffée de citations littérales du texte proustien qui se trouve dans des cadres bien distingués. En plus ces citations se particularisent par la couleur de fond: jaune d'œuf précise le commentaire. L'effet est spécifique : l'album présente un résumé express de la Recherche composé de 
citations clé, de passages choisis comme étant des fragments essentiels, pour le contenu autant que pour le style (nombreux sont par exemple les endroits où des séries d'adjectifs se concatènent proustiennement). Recueil de citations vedettes présentées sur un plateau, traversée rapide de l'œuvre pour une première dégustation. On peut évidemment reprocher à une telle facture de négliger l'élément majeur de la Recherche qu'est le Temps ; pour un lecteur patient et attentif l'initiation que constitue l'errance du texte se célèbre par définition dans le temps et par le temps. Précisons d'ailleurs que -cette négligence de la durée étant probablement inévitable si on ne se constitue pas adepte de Pierre Ménard - cette forme courte du roman, qui paraît s'inspirer plutôt des réflexions de Pierre Bayard ${ }^{3}$, semble bien vouloir suivre deux autres principes de Proust auteur à savoir les effets de rythme et les manipulations de la fréquence.

Nulle part le goût du théâtre apparaît chez Proust avec autant de force que dans son emploi de la scène romanesque, pourvue d'ordinaire de prologue, de coup de théâtre et d'épilogue. On pourrait dénombrer quelques centaines de ces scènes dans la Recherche; le reste se compose de réflexions, de méditations, de ruminations, de rêveries et de descriptions qui donnent à ces scènes leur gîte et leur poids. Dans la version bd cet aspect de la Recherche est encore plus accentué; dans ce sens-là il s'agit donc d'une vision particulière (et en principe justifiée) de l'œuvre. La bande dessinée s'est diversifiée de telle manière que de multiples procédés peuvent se marier avec une pléthore d'intentions. Il est indéniable néanmoins qu'elle paraît se prêter fort bien à la représentation de scènes 'théâtrales', possibilité exploitée visiblement dans le cas présent. Ainsi le premier album des Jeunes Filles s'est construit autour des scènes suivantes: le voyage en train et l'arrivée à Balbec; l'installation au Grand Hôtel; la rencontre de Mademoiselle de Stermaria ; faire la connaissance de Madame de Villeparisis; excursions à la campagne (arbres d'Hudimesnil); arrivée de Saint-Loup; frasques de Bloch; premiers contacts avec Charlus; apparition des jeunes filles. Quelques planches intercalées présentent surtout le paysage et son architecture. Pour les autres albums on trouve une répartition comparable. On remarque que les scènes se situent avant tout autour de personnages : et c'est une des caractéristiques majeures de la bd de se construire à base de personnages. 
Pour ce qui regarde la fréquence, Gérard Genette a bien dégagé cette tendance majeure de l'œuvre proustienne qui est de présenter en tant que habitude, coutume, scénario répétitif ce qui à force de détails et de spécifications ne saurait avoir lieu qu'au niveau de l'unicité. C'est le pseudo-itératif qui donne aux scènes leur incrustation particulière, leur goût de déjà vu, leur familiarité, voire leur étrangeté familière. Dans la bande dessinée cette itérativité se concrétise dans la répétition des figures et de leur décor où souvent tel aspect symptomatique oriente immédiatement le repérage. Dans le cas de Heuet deux éléments parallèles renforcent cette stigmatisation : la nature simple des traits, c'est-à-dire qu'il y a relativement peu de détails, et le côté statique (les mouvements se placent entre les images plutôt qu'à l'intérieur). L'impression est en effet celui d'une projection de diapositives comme pour la lanterne magique. Ceci peut répondre dans un sens à l'intention de Proust : les changements et le dynamisme se situent à l'instant des découvertes fulgurantes, des transitions brusquées, des coups de théâtre et des coups de foudre. Pourtant une autre conséquence est moins conforme au message de la Recherche : la caractérisation des personnages.

\section{Textes}

Mais avant d'en venir à eux il convient de donner encore quelques précisions sur la dimension textuelle. La prose de Proust se retrouve de trois manières: comme récitatif (les citations encadrées à part); comme dialogue (reprise du texte avec certains raccourcis) et comme transfert en images. La spécificité de la bande dessinée se détectera surtout pour ces derniers cas. On y reviendra mais notons tout de suite qu'ils sont relativement peu nombreux (ce qui donne d'autant plus de poids à ceux qui subsistent).

Les récitatifs occupent régulièrement trois à six bandes horizontales par page ; leur présence est relativement discrète mais incontournable, et ils attirent le regard avant tout autre élément, guidant donc la suite de la lecture. Parfois pourtant ils envahissent tout l'espace ou presque, ainsi Combray page 40 au sujet de Bergotte: comme d'ordinaire la page se 
compartimente en trois couches à quoi s'ajoutent deux bandes horizontales sur toute la page. Nous avons ainsi 11 vignettes dont 7 sont entièrement remplies de récitatifs, alors que trois autres comportent en plus un cadre intérieur consacré au récitatif. Et ce n'est pas tout car la deuxième vignette montre des pages d'un livre superposées (des pages de Bergotte peut-on supposer $^{4}$ ) et la troisième se gonfle considérablement de citations bergottiennes. Le garçon lecteur au centre de la page est visiblement englouti par tout cet amas de texte. Ici la bande dessinée se fige en tombeau ; la pétrification arrête tout dynamisme.

Heuet a pu s'apercevoir des risques d'une trop grande invasion citationnelle : c'est pourquoi sans doute il a parfois tenté d'évacuer le texte pour telle ou telle scène, mais en outre il introduit régulièrement des éléments dessinés au sein même des cases réservés au récitatif. Ce sont des silhouettes découpées ou des ombres chinoises qui constituent une sorte de transition entre une édition de la Recherche pourvue d'ornementation (comme celle de l'éditeur Jean de Bonnot) et la bande dessinée proprement dite $^{5}$. Quand ces figures restent ornementales (comme les aubépines, page 47), la transition entre les vignettes en est facilitée, mais en y introduisant des figurines (comme pour Combray page 41 où le texte du récitatif: "Quand j'eus appris ce jour-là que Mlle Swann était un être d'une condition si rare, baignant comme dans son élément naturel au milieu de tant de privilèges,... » est accompagné d'une petite silhouette en noir de poupée enrubannée) l'auteur risque de trop détourner l'attention.

Dans Les jeunes Filles cet art des silhouettes se transforme pourtant et privilégie les petits groupes qui passent: de la sorte un certain dynamisme s'infiltre dans les inscriptions. Prenons comme exemple le groupe des six jeunes filles qui passent en sautillant (II 12) : ainsi se transmet très bien la notion de passage, de transition éphémère, de fugacité qui adhère à elles et qui scellera jusqu'au bout la relation avec Albertine.

Pour la relation entre récitatif et dessins regardons encore la toute première page de Combray. Comme dans les livres d'antan un long L plié survole le cadre initial où se dégage le texte attendu: "Longtemps, je me suis couché de bonne heure», coiffant le dessin d'un volet clos ouvrant paradoxalement (et rousselliennement) le récit. Vu que la partie supérieure de cette page est occupée par le titre (première partie : COMBRAY), on ne 
trouve que deux strates de dessins. Chaque vignette est surmontée d'un récitatif encadré. On remarque une nette progression: chacune des trois vignettes du haut s'élargit comparée à la précédente: c'est le réveil nocturne (2) où l'univers est sens dessus dessous, sensation rendue ici par des bandes transversales bleu/blanc donnant une certaine giration à l'ensemble ; c'est l'arrivée du souvenir (3) qui fait tourner les éléments de la chambre alors que les deux yeux du dormeur éveillé surplombent la scène. La couche d'en bas est nettement plus haute (« ...le branle était donné à ma mémoire...») et comporte deux vignettes plus larges aussi: dans la première les yeux dominent la mer qui est entourée de sites divers : Paris, Balbec, Doncières, mais aussi un avion, une fenêtre, un zeppelin au centre de rayons lumineux. C'est ensuite la cinquième vignette qui complète cette vague de souvenirs sur fond de Venise alors que les yeux ont disparu. Ainsi on se trouve au milieu d'un condensé de la suite de la Recherche (ce qui est bien présent dans le texte de Proust mais de façon moins exclusive), tableau d'ensemble où le figuratif et l'imaginaire se rejoignent. Le bleu en est la couleur dominante, ce qui semble pertinent : chez Proust le bleu est la couleur maternelle comme origine (du désir, du monde). À la page suivante les couleurs criardes des planches de la lanterne magique vont interrompre cette entrée nocturne et aquatique, cette (re)traversée des eaux amniotiques. 


\section{Marcel Proust \\ Dessins de Stéphane Heuet}

\section{À la recherche du temps perdu}

À l'ombre des jeunes filles en fleurs

VOL U ME I

$$
\text { के } 2 x^{2 x}
$$

(1)

DELCOURT

couverture Jeunes Filles 1 


\section{Personnages}

L'élément central de la bd est bien constitué par le personnage qui est au noyau de la diégèse et qui est essentiellement répétitif. Nous savons que la répétitivité et le retour des personnages est également un facteur capital pour Proust. Marie-Hélène Gobin a pu noter que le jeune Marcel hérite ses caractéristiques principales de Tintin et que Françoise peut être rapprochée de Bécassine ${ }^{6}$. Ces conclusions peuvent s'étendre à d'autres personnages comme Gilberte, Albertine, Charlus, Saint-Loup et dans Un Amour de Swann les Verdurin, Cottard ou Swann lui-même par exemple. Il s'agit d'un choix bien précis parmi les différentes formules qui se croisent, se superposent et s'enchevêtrent chez Proust. Le dessin à la manière d'Hergé avec ses figures bien délimitées, à contours précis, pourvues d'un nombre restreint de traits caractéristiques, répond à une première vision fondamentale de Proust (surtout orientée dans ce cas-là par le regard du novice comme en témoignent les planches de la lanterne magique) : c'est le côté symptomatique des personnages. Pourtant Proust ne tarde pas à compliquer cette galerie : si les comparses conservent tout au long de leur carrière livresque leurs tics et leurs gestualité de marionnettes (ainsi le directeur de l'hôtel de Balbec, ce dont profite pleinement Heuet), les protagonistes se distinguent par toute une série de transformations et d'autre part par un flou qui les entoure, une corporéité fluctuante et dynamique, une mimique ambigüe et un comportement souvent mystérieux. Ceci peut trouver son origine dans les intermittences de la vision ou du cœur car l'autre est tel qu'on le désire suivant les métamorphoses du désir même. Une autre raison des transformations et du degré d'incertitude dans l'appréhension des personnes peut se trouver dans leur nature d'artiste : là réside le fond d'Elstir, de Vinteuil, d'Octave, de la Berma. Heuet n'a pas essayé de rendre cette deuxième dimension qui constitue un pilier important de la psychologie de Proust, ainsi que de son esthétique (proche de celle de Duchamp ici, qu'on pense à « Nu descendant $\mathrm{d}^{\prime}$ un escalier ») et de sa réflexion philosophique. Une petite excursion dans ce sens ainsi qu'en témoigne le portrait d'Albertine ne peut pas vraiment 
convaincre. La fameuse tache qui se déplace suivant le souvenir de Marcel et qui annonce la future insaisissabilité de la jeune fille ne saurait rendre la fugacité de la vie à un dessin simplifié. Si Proust conserve bel et bien cette même dimension enfantine dans son œuvre, naïvement parfois, ironiquement plus souvent (avec les caricatures bienveillantes ou féroces allant des grand-tantes à Legrandin, de Françoise à la tante Léonie, d'Odette à Madame de Guermantes) il y superpose toujours d'autres dimensions qui signent la complexité de la Recherche.

Et Marcel (servons-nous de ce prénom pour faciliter l'analyse) est le personnage le plus complexe de tous vu surtout qu'il y a une dimension narratrice qui à la fois intègre, réunit, amalgame, confond, redistribue et totalise les morceaux, les avatars, les phénomènes, les apparitions, les déguisements, les formes théâtrales, ludiques, mensongères, artistiques, du JE. Marcel est le petit garçon en costume marin, il est aussi l'adolescent curieux et frileux, il est le jeune adulte amoureux et jaloux, il devient l'adulte déçu et mondain, il sera l'écrivain tout voué à son art. Mais surtout il est tout cela à la fois dans la Recherche, car c'est lui-même qu'il cherche, son temps à lui et sa vocation, vocation on le sait qui se confond avec notre lecture. La Recherche de Heuet est donc fidèle au texte mais dans une version pour enfants qui évite (nécessairement?) les affres et l'euphorie de la perte constitutionnelle de soi.

\section{Tressages}

Groensteen (o.c.) a raison selon nous d'accentuer dans sa définition de la bande dessinée la primauté de la macrostructure dont dépend la microstructure, de souligner l'importance des combinaisons, de ce qu'il appelle l'arthrologie (les connexions à différents niveaux) et de la dimension du tressage (le retour de certains éléments comme refrains ou comme échos). Et c'est le lecteur qui accomplit ce travail de combinaison, qui (re)crée le plein entre les images.

Dans la relation entre le texte de Proust et la bande dessinée un vrai défi pourrait être de trouver une formule bd pour ce phénomène capitalissime qu'est le tressage pour l'auteur de la Recherche. Chez lui la 
reprise est essentiellement le fruit de la différance, ce qui se répète est autre par là-même et par la distanciation spatio-temporelle inhérente. Le retour de l'identique est l'utopie de l'extemporel, si férocement haussé sur le piédestal de l'idéal qu'il ne peut que s'effondrer. Mais ce tressage a aussi sa valeur artistique : ce qui dans les êtres se diffracte et se dissémine peut se coaguler dans l'œuvre d'art, même si ce n'est que sur le ton de la mélancolie. Il est vrai que ce genre de manifestations se révélera surtout dans les volumes de la Recherche que Heuet n'a pas encore abordés. Mais si on regarde le retour de Charlus (entre Combray et Les Jeunes Filles), la transformation de Biche en Elstir, le passage de Miss Sacripant à Madame Swann, l'évolution de la figure d'Albertine suivant les cristallisations et les pétrifications de l'amour, ainsi que les avatars du 'je', on s'aperçoit que la bande dessinée reste unidimensionnelle là où le texte se fissure et se réencastre continument.

Pourtant si sur ces deux points (la mobilité du personnage et les effets de construction à distance) les intentions de la Recherche n'ont été que peu exploitées jusqu'ici par Heuet, il y a un certain nombre d'endroits dans les différents albums qui témoignent d'une véritable recherche formelle. Examinons-en quelques-uns pour juger de leur pertinence.

\section{Combray}

Combray, le premier album qui fonctionne comme matrice de la série, suit assez fidèlement le texte de Proust. La vie familiale à Combray avec les visites de Swann, l'oncle Adolphe, Françoise et la Charité de Giotto, la lecture dans le jardin, les promenades, la découverte de Bergotte, les scènes à Montjouvain, le 'geste' indécent de Gilberte en constituent quelques étapes attendues. $\mathrm{Vu}$ que tout novice dans l'univers de la Recherche se voit d'ordinaire régalé d'une bonne bouchée de madeleine au parfum de fleur d'oranger, regardons de plus près si la bande dessinée réussit à en faire un pareil générateur. Nous pouvons constater que le mouvement qui accompagne le surgissement des données essentielles de la diégèse (l'arrivée des souvenirs) profite de la mise en espace de la bd. En effet si la première orientation de la bd repose dans la succession des images, leur 
concaténation, et que suivant la définition de Groensteen elle « est un mode d'expression séquentiel, caractérisé par la juxtaposition d'une pluralité d'images solidaires", on peut détecter d'autre part un mouvement de verticalité (étroitement lié au principe de la succession) qui dépend d'abord de la manière dont la lecture 'descend' la page, mais qui insiste aussi dans l'exploitation de la troisième dimension - la profondeur de l'image. Le geste de la verticalisation comme originel mouvement d'art a été fort bien dégagé par Gilbert Durand ${ }^{7}$ et pour ce qui concerne Proust, Malcolm Bowy $^{8}$ a révélé dans une fascinante étude que la verticalité au-delà d'une attitude anthropologique et mythique s'enracine dans les paradigmes de la connaissance du $19^{\mathrm{e}}$ siècle (archéologie, historiographie, philosophie). Cette mise en valeur de la verticalité chez Proust restera une constante tout au long de la Recherche même si elle sera enrichie par tout un ensemble de mouvements rhizomateux. On la repère notamment lors de moments charnière comme la description du clocher de Combray, la levée du jour, la montée à la chambre ou inversement la descente dans les cryptes. Cette tendance trouve un de ses instants privilégiés lors de la montée des souvenirs que ce soit par ingurgitation de pâtisseries ou encore en valsant sur des pavés inégaux.

Cette fondamentale et génératrice verticalité, la bande dessinée peut en procurer sa propre version imagée. On trouve chez Heuet des représentations variées de cette dimension plastique, que ce soit par l'image de la montée vers la chambre inaugurant le 'drame du coucher' (13), par celles de la tour de l'église (22 entre autres), par la descente à la crypte (21), ou encore par l'effet de la pluie vu d'en haut (en plongée donc). C'est d'ailleurs régulièrement par des vues d'en haut que l'effet de verticalité s'instaure ou s'accentue, ainsi pages 5 et 9 (le cercle familial vu d'en haut insistant sur son homogénéité) ou des panoramas du village (ce petit univers clos, 45).

Dans le cas de la madeleine la matérialisation du passé se réalise d'une manière semblable. À la page 14 nous reconnaissons l'homme emmitouflé de la couverture annonçant ainsi la 'découverte' imminente. Il entre chez maman et elle lui présente le petit gâteau dodu. De la petite cuiller qui semble planer dans l'air commencent à monter les salutaires vapeurs, tandis que le réel (fort prosaïquement figuré par la théière) 
s'efface et que le textuel se met à envahir l'espace («ceci n'est pas une madeleine »), précédé d'un point d'interrogation en plein centre de la page 15. La descente de la page s'inverse finalement parce que la dernière image remonte vers le haut suivant la volute vaporeuse et parfumée (qui se prolonge en épousant la mèche du personnage lequel extatiquement ferme les yeux et lève le visage). Il y aurait une longue analyse à faire de la relation ici entre récitatif (doublé), monologue intérieur extériorisé et dessin. Le monologue, lui (accompagné de petits points et de guillemets), serpente sur le visage en suivant les courbes de la joue et du front (mais « la messe » se perd dans un sourcil) : « ... bientôt l'heure de la messe...» et «bonjour, tante Léonie... »; le récitatif, dont le premier tronçon parle en haut de "ce qui palpite au fond de moi », curieusement se termine en bas par les mots : "Arrivera-t-il jusqu'à la surface de ma claire conscience, ce souvenir, l'instant ancien...». On s'aperçoit que la tension entre la succession textuelle (symbolique) et la tendance iconique à la verticalité se pourchassent. La lecture déconstructrice qu'on entame ici montre leur rivalité épistémologique et déontologique ${ }^{9}$, mais surtout leur concurrence technique et matérielle. Ce qui nous permet de tourner la page... où la première vignette est une complète montée sur toute la hauteur de l'espace disponible: successivement nappe, théière, tasse, Marcel en transe gustative, spectrale ascension des buées et répétition du texte « ... bonjour, tante Léonie... ${ }^{10}$ arrivent à un ciel mordoré qui ne dénote donc nul extérieur mais l'univers textuel où repose la couchée sempiternelle. Deux autres vignettes de cette page montrent l'une au-dessus de l'autre la chambre de la tante et la place de Combray (avec Marcel qui accentue la ronde des images et des trainées mémorielles en manipulant son cerceau). Par transition textuelle (un récitatif qui chevauche la double page) cette entrée ouvre sur une page pleine qui exhibe le village en image d'Épinal, décor de conte de fée encadré d'aubépines et de nymphéas, bordé ensuite de verdure, et où la couronne des maisons finalement entoure l'église Saint Hilaire. Visuellement l'arthrologie se fait par les bandes nuageuses provenant de la tasse, et alors que le récitatif se superpose comme une sorte de table de la loi à l'idyllique et arcadien locus amoenus, ces pseudophylactères à espace disponible, virtuellement inscriptible, s'envolent gracieusement vers la blancheur des marges, promettant la suite du récit et 
la succession des images (dont la première tout en haut de la page 18 ne manque de figurer le temps). Le village sera ensuite surtout une totalité homogène où l'enfant (et le narrateur) retrouvent unité, totalité et simultanéité intimement désirées. Un ultime exemple serait constitué par la page 27 où les plaisirs de la table s'articulent sur une pleine page autour d'une Françoise glorieuse.

\section{À l'ombre des jeunes filles en fleurs}

Dans À l'ombre des jeunes filles en fleurs 1 les mesures de l'espace sont particulièrement fouillées. C'est surtout le Grand Hôtel de Balbec qui permet au dessinateur d'impressionner la page. Page 13 sa vaste fenêtre domine la plage ; c'est de là-haut qu'on prend possession du monde. La double page 20-21 permet de contourner le vaste édifice le soir, d'entrer d'abord dans la salle à manger parmi les privilégiés et de diriger le regard ensuite vers les gens du village qui reluquent bouche bée cette scène du dehors. La bd est un art de l'espace compartimenté et permet de tirer des effets particuliers de cette position. Ici la place du Grand Hôtel comme plaque tournante de l'ordre séquentiel et comme plateforme où les mentalités et les attitudes des différents personnages alternent est valorisé de manière intéressante. Pour le personnage de Marcel l'hôtel est tout d'abord un lieu inconnu où les habitudes le délaissent cruellement. La chambre au vétiver à la page 10 se trouve au bout d'un escalier vertigineux et par ses dimensions étirées se met à valser elle-même. Ensuite une vue en plongée montre le garçon dans son lit, perdu dans un univers hostile, sans véritable point d'attache. Le second volet de la double page permet ici d'introduire de façon spécifique le fort contraste que Proust vise par d'autres moyens. C'est que le lendemain la fenêtre s'ouvre sur la mer, «la mer nue ", qui va remplir tout l'espace avec le vent et les nuages, directement ou encore par leur reflet dans les vitrines; c'est l'interpénétration de la terre et de la mer («collines de la mer », préfigurant l'expérience avec Elstir) qui permet cette métaphore-métamorphose salvatrice. 


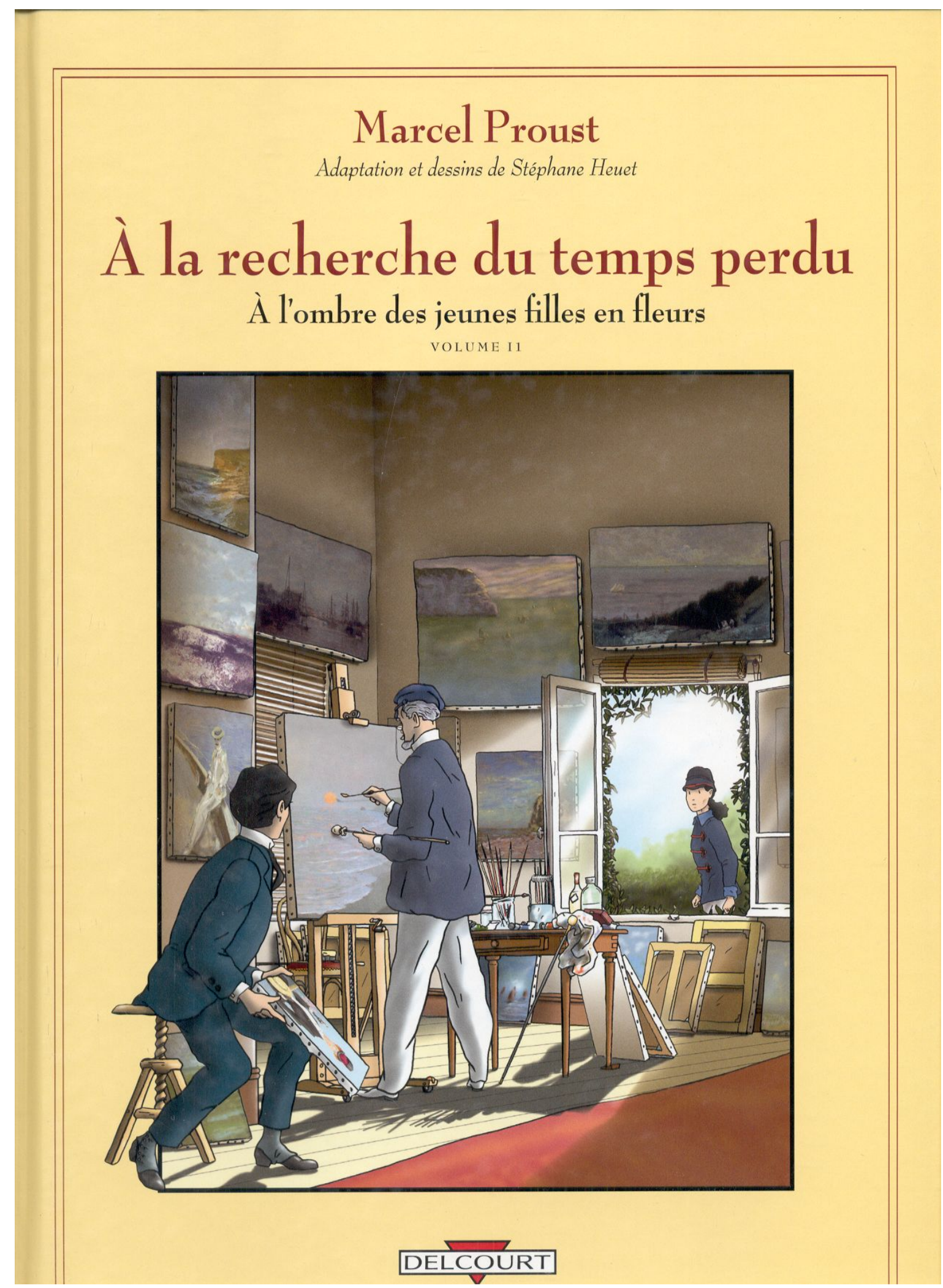

couverture Jeunes Filles 2 
Autre effet (opposé) : celui de la double page 26-27. Page 26 raconte la déception quand pendant une excursion dans la campagne normande les arbres d'Hudimesnil ne répondent pas au désir de l'extratemporel qu'ils semblent renfermer : ainsi s'ouvrira ce vide artistique et existentiel dont Marcel va tant souffrir. Page 27 tente de restituer cette angoisse. Toute trace de texte a disparu et les couleurs ont viré aux teintes foncées où domine le pourpre funéraire; on est en plein imaginaire et au-delà de tout principe de plaisir; la voiture s'enfonce vers un néant de Götterdämmerung et glisse dans la nuit comme un vrai corbillard (ce qui se prolonge encore un peu à la page suivante avant de se dissiper dans les lumières de l'hôtel retrouvé). Cet espace (dessiné) où la voix a été suspendue se détache comme spécifiquement efficace.

Un autre aspect que cet album exploite à plusieurs reprises est le côté 'multimédia' que rend possible la bd ${ }^{11}$. Ainsi la façade de l'église de Balbec est combinée page 8 avec des représentations des moulages et avec des images dans un livre; sur la page précédente on pouvait voir aussi un vitrail et ses différentes 'cases'. D'une certaine manière on comprend la déception de Marcel dont l'imagination avait pris un haut vol. C'est «la tyrannie du particulier » : ainsi la bande reconnaît dans un sens sa propre défaillance (provisoire). On n'est pas étonné de voir apparaître un petit supplément sur la porte de l'église: une affiche invitant à voter sur tel homme politique.

Un autre exemple du même procédé se trouve vers la fin de l'album où le personnage de Charlus est présenté en deux temps : d'abord suivant les histoires de Saint-Loup qui fait de son oncle un portrait de grand charmeur, ce qui résulte dans la pensée de Marcel dans une image copiée sur Robert de Montesquiou tel que nous le montre le tableau de Giovanni Boldini du Musée d'Orsay. Le Charlus 'réel' qui surgit ensuite à Balbec 'retombe' dans le genre des comics: l'impression étrange qu'il fait sur Marcel (dans le texte de Proust) et dont celui-ci ne découvrira que beaucoup plus tard que c'est le symptôme de l' «inversion», devient ici franchement loufoque. Charlus s'enfonce entre l'idéalisation de certains lecteurs «à clefs » (le Montesquiou) et une espèce de Capitaine Haddock. Le vrai Charlus se faufile entre les hortensias omniprésents et différentes 
suggestions érotiques que le lecteur peut apprécier. ${ }^{12}$ Cette présenceabsence d'un personnage rend pourtant plutôt bien la tendance proustienne omniprésente de va-et-vient constant entre des portraits fabuleusement enrichis par l'imagination et des observations réalistes réduites à des traits élémentaires. Les intermittences de la vue, suivant sa direction intériorisée ou directe, interfèrent inextricablement avec les intermittences du cœur. Seulement: si le lecteur de la bd remplit les vides entre les images, celui qui se plonge dans le texte littéraire crée beaucoup plus généralement son propre univers ainsi que le précise Le temps retrouvé. 


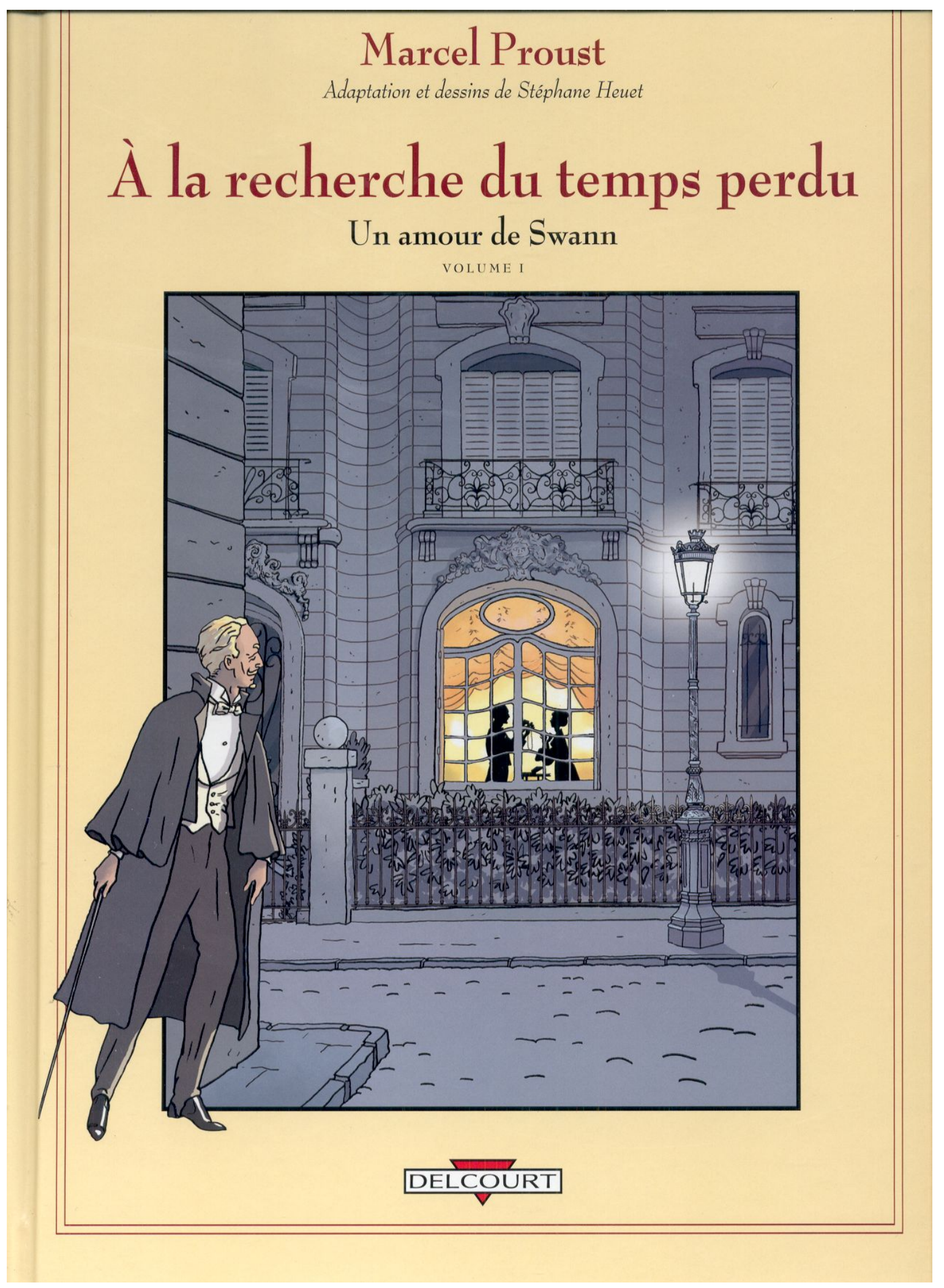

couverture Amour de Swann 1 


\section{Un Amour de Swann}

Un dernier exemple concernera Un Amour de Swann où d'ailleurs revient le jeu avec l'intermédialité au sujet du fameux portrait de Zéphora qui fonctionne en tant que catalyseur pour l'amour de Swann. Heuet signe et persiste dans le vice de Swann consistant à concrétiser l'art dans la vie (il ajoute d'autres tableaux qui ne sont pas directement indiqués par Proust, page 28), ce qui permet une comparaison entre les genres ici (bd et tableau) indiquant au moins leur altérité. Un premier exemple de cette pratique se trouve déjà dans Combray pour les allégories de Giotto.

L'amour et l'art se combinent de bien d'autres manières encore dans le récit de Swann. Deux passages sautent aux yeux plus particulièrement: le rôle que joue la sonate de Vinteuil en tant que hymne national de leur amour et la littérarisation de l'acte physique correspondant dans l'expression « faire catleya ».

Une large double page (18-19) permet à la musique de se visualiser après une mince bande d'ouverture qui relate la première écoute. Le résultat peut se définir comme poly-imagé : $s^{\prime} y$ croisent et $s^{\prime} y$ superposent des récitatifs (citations), des vignettes dessinées montrant Swann pendant la deuxième écoute (se résumant au milieu à une mince tranche de sa figure), et un fond d'aquarelle étalant un vaste paysage - un lac entouré de montagnes ainsi qu'une roseraie sur le devant avec trois fenêtres illuminées en coulisse. Devant la partie supérieure de ce paysage on voit dans l'air des rubans remplis de notes, larges guirlandes musicales évoluant comme une traine de robe de mariée, alors que tout en haut on aperçoit également une partie des deux instruments de musique (le violon et le piano). Des deux côtés les lignes musicales débordent légèrement le cadre général et elles établissent de la sorte une ligne de continuité, une fluidité esthétique rejoignant le motif proustien de l'eau vivante. La synesthésie artistique qui est ainsi suggérée apparaît comme un essai certes insuffisant, mais néanmoins suggestif de donner une image de l'importance de la synesthésie proustienne, cette métaphore des sens et des sensations qui loin avant toute intellectualisation dirige la Recherche. 
L'affaire des catleyas se situe comme contrepoids après une première occurrence de l'angoisse de perdre Odette (page 33 sq.). La partie suggestive précède plutôt celle des caresses proprement exécutées (c'est une petite traversée de Paris à la façon du fiacre conduisant Emma Bovary et son amant par les rues de Rouen). L'érotisme de ces vignettes est fort restreint, et c'est comme si le dessinateur avait senti le besoin de remédier à cette faiblesse constitutionnelle de sa manière. Ce sera d'abord par un recours aux dessins de Watteau (36) dont Proust fait également mention et qui ébauchent des sourires comme ceux d'Odette. Si la valeur artistique est discutable, l'intention de rendre ainsi la fugacité de l'être est intéressante. Pourtant l'image toute heuettienne qui clôt cette série est celle qui occupe la majeure partie de la page 37. Odette est étalée sur le divan et entourée $\mathrm{d}^{\prime}$ une vaste bibliothèque à plusieurs étages où Swann la considère d'en haut. Il est parfaitement clair qu'elle n'est nullement une femme de livres mais entièrement un être livresque. " $\grave{A}$ suivre»: ainsi se termine verbalement l'album où la véritable chaîne arthrologique se dégage pourtant d'une ultime fleur de catleya d'où paraissent émaner en pointillé des saveurs inédites. 


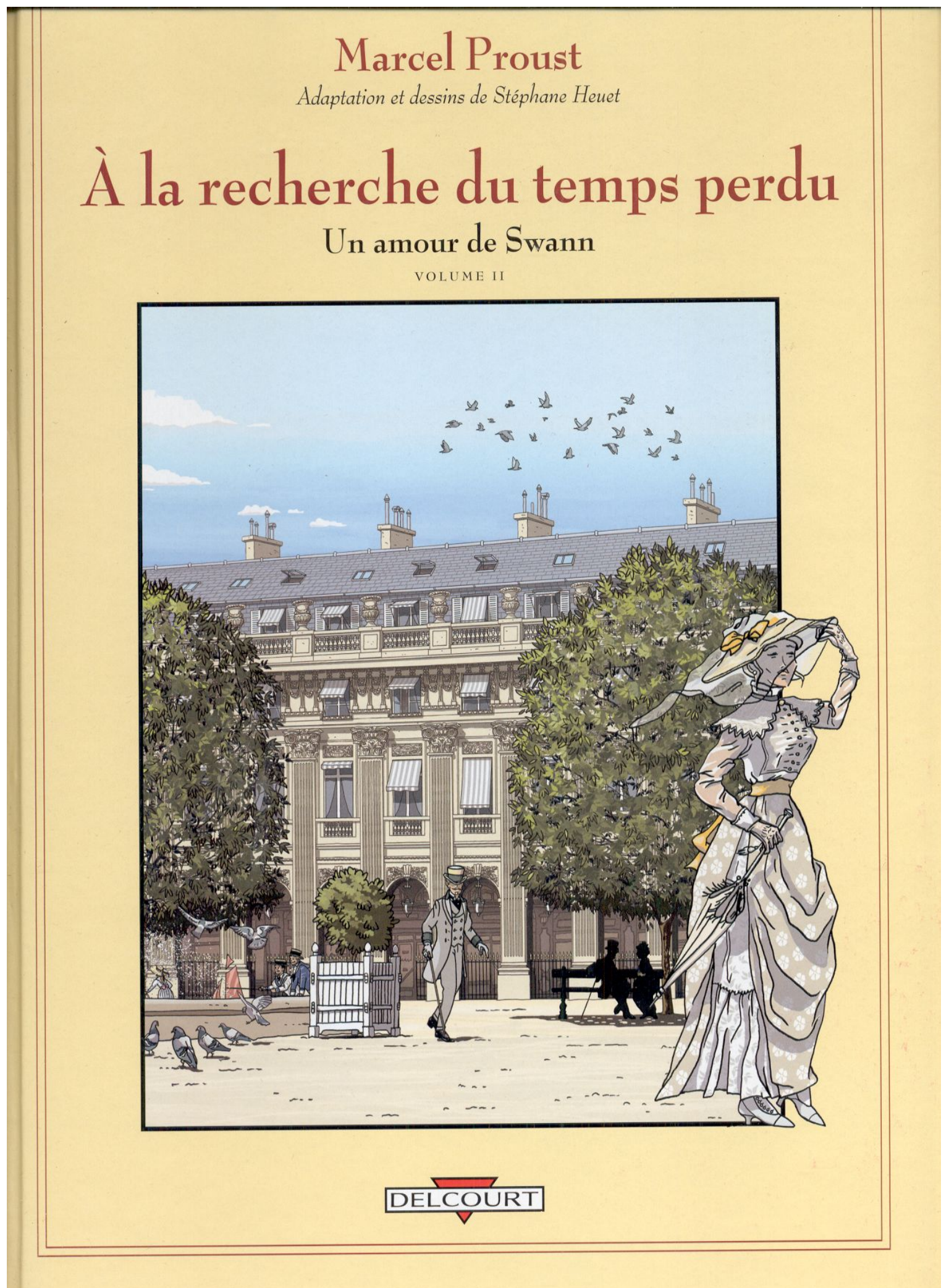

couverture Amour de Swann 2 


\section{Notice}

Sjef Houppermans enseigne la littérature française moderne à l'université de Leiden. Il a publié en 2007 chez Rodopi Marcel Proust constructiviste. Courriel : j.m.m.houppermans@let.leidenuniv.nl

\section{Notes}

${ }^{1}$ Pour les termes techniques ainsi que pour les apports théoriques et méthodologiques nous nous sommes servi du Système de la bande dessinée de Thierry Groensteen (PUF, 1999).

${ }^{2}$ Il est curieux que tandis que pour Combray est mentionné sur cette page le titre Du côté de chez Swann, c'est-à-dire celui du livre 1 de la Recherche dont Combray constitue le premier volet, dans le cas d'Un Amour de Swann (le second volet) ce titre manque.

${ }^{3}$ Le hors-sujet, Proust et la digression, Minuit, 1996.

${ }^{4}$ Le lecteur attentif remarquera qu'il s'agit en fait de la copie de la fameuse lettre de Mallarmé à Verlaine datant du 16 novembre 1885. Dans cette lettre autobiographique Mallarmé parle du Livre comme projet de toute sa vie et le passage suivant notamment peut se décrypter dans l'album de Heuet : " Rien de si simple alors que je n’aie pas eu hâte de recueillir les mille bribes connues, qui m’ont, de temps à autre, attiré la bienveillance de charmants et excellents esprits, vous le premier! Tout cela n'avait d'autre valeur momentanée pour moi que de m’entretenir la main : et quelque réussi que puisse être quelquefois un des morceaux ; à eux tous c'est bien juste s'ils composent un album, mais pas un livre. Il est possible cependant que l'Éditeur Vanier m'arrache ces lambeaux mais je ne les collerai sur des pages que comme on fait une collection de chiffons d'étoffes séculaires ou précieuses. Avec ce mot condamnatoire d'Album, dans le titre, Album de vers et de prose, je ne sais pas; et cela contiendra plusieurs séries, pourra même aller indéfiniment, (à côté de mon travail personnel qui je crois, sera anonyme, le Texte y parlant de lui-même et sans voix d'auteur). »

5 Alors que le premier exemple en concerne la fille de cuisine enceinte (Combray page 34), significativement la figure de Bergotte sur la planche de la page 40 fait migrer un semblable emblème à l’intérieur du récit.

${ }^{6}$ In Proust et la bd? Que dira Baudelaire ; étude sémiotique, Éditions Connaissances et Savoirs, 2006. M-H Gobin consacre surtout son travail à une étude précise des modalités de l'énonciation.

${ }^{7}$ Les Structures anthropologiques de l'imaginaire, Paris, Dunod (1 $1^{\text {re }}$ édition Paris, P.U.F., 1960).

${ }^{8}$ Freud, Proust and Lacan, Theory as Fiction, Cambridge University Press, 1988.

9 La majeure transition historique et scientifique étant celle entre des systèmes verticaux (théotéléologiques) et des modèles horizontaux (technico-immanentistes).

${ }^{10}$ Cette phrase ne figure d'ailleurs nulle part dans le texte de Proust sous sa forme de discours direct.

${ }^{11}$ C’est cette multimédialité utilisée afin de représenter la stratification plurielle de la Recherche qu'a employée de manière très réussie le metteur en scène flamand Guy Cassiers dans ses versions théâtrales de l'œuvre de Proust réalisées pour le RO théâtre de Rotterdam (2002-2006).

12 Alors que les albums en sont pleins, on ne compte chez Proust que deux occurrences de ces fleurs au signifiant prometteur (hors temps il y a...) : des hortensias roses - par rapport à la Normandie d'ailleurs dans Le Temps retrouvé et des hortensias bleus dans le premier tome. Les hortensias bleus est également un titre de Montesquiou. 